\title{
Continuous Monitoring of a Transesterification Reaction Using an Analytical Balance
}

\author{
Matthieu Tubino, * Arnaldo G. Oliveira Júnior and Acacia A. Salomão \\ Instituto de Química, Universidade Estadual de Campinas, P.O. Box 6154, 13083-970 Campinas-SP, Brazil
}

\begin{abstract}
An electronic analytical balance was used to monitor a transesterification reaction through density variations. To test the proposed system, we studied how the transesterification reaction was influenced by: (i) the addition of methanol to the oil; (ii) the addition of the catalyst; (iii) stirring; and (iv) temperature.
\end{abstract}

Keywords: transesterification reaction, continuous monitoring, analytical balance

\section{Introduction}

Biodiesel is an alternative fuel to diesel derived from petrol. It is prepared via transesterification of vegetable oils and animal fats, rendering it a green fuel source. ${ }^{1}$ The transesterification reaction generates a mixture of alkyl esters, methyl or ethyl, depending on the type of alcohol used, methanol or ethanol. It has physicochemical properties similar to petrodiesel and, therefore, it can be used directly in diesel engines. ${ }^{2}$ Because it is prepared from biodegradable raw materials it has advantages over common diesel fuel such as the absence of sulfur in its emissions, a high flash point, and a good lubricity. ${ }^{3,4}$

The physicochemical characteristics of biodiesel are quite different from those of vegetable oil, a fact that is used in the present work in order to study the transesterification reaction. Previous studies have monitored this reaction by removing samples from the reaction mixture at different time points. ${ }^{5}$ However, real time monitoring of the transesterification reaction is desired. This can be accomplished, for example, using infrared monitoring. ${ }^{6-8}$ Recently, the refraction index was used to study the mechanism of a transesterification reaction by this method. ${ }^{9,10}$

With the aim to develop a simple procedure for monitoring this reaction in real time so that more detailed investigations about the transesterification reaction can be conducted, the feasibility of using an analytical balance was studied. The results from this method are very interesting and are described below.

\footnotetext{
*e-mail: tubino@iqm.unicamp.br
}

\section{Experimental}

\section{Materials}

Reactor: a 2 L round bottom glass flask equipped with a mechanical agitator (OS20-5 Lab 1000), coupled to a glass stirring rod with a Teflon helix; peristaltic pump: Gilson Miniplus $3^{\circledR}$; pumping tubes: Tygon ${ }^{\circledR}$ 2-Bridge (Red-Red) PVC Solva tubing ${ }^{\circledR} 1.14 \mathrm{~mm}$ internal diameter; conducting tubes: polyethylene $1.0 \mathrm{~mm}$ ID; phase separators: made of poly(methyl methacrylate); analytical balance: Adventurer Pro Ohaus equipped with an RS 232 interface and the 80850080 Software WinWedge; electronic thermometer: Instrutherm THR-080 with a Pt-100 sensor; laptop: computer to receive and analyze the data, Origin ${ }^{\circledR}$ SR3 v8.34.90 software to analyze the data; optical microscope: NIKON E800, 40× objective; stage micrometer: TYPE A MBM11100 Nikon $0.01 \mathrm{~mm}$.

\section{Reagents}

Refined soybean oil was purchased from the local market. Methanol was analytical grade (Synth Diadema, São Paulo). A 30\% sodium methoxide solution (m/v) was analytical grade (Vetec, Rio de Janeiro, Brazil). Food coloring from Arcolor (Brazil) is a mixture of Red IV (Ponceaux) and Red III (Bordeaux).

\section{System set-up}

The monitoring system was assembled according to the scheme shown in Figures 1 and 2. Special attention was paid to the connections between tubes as well as between 
the tubes and the other pieces, such as the phase separators, in order to avoid leaks.

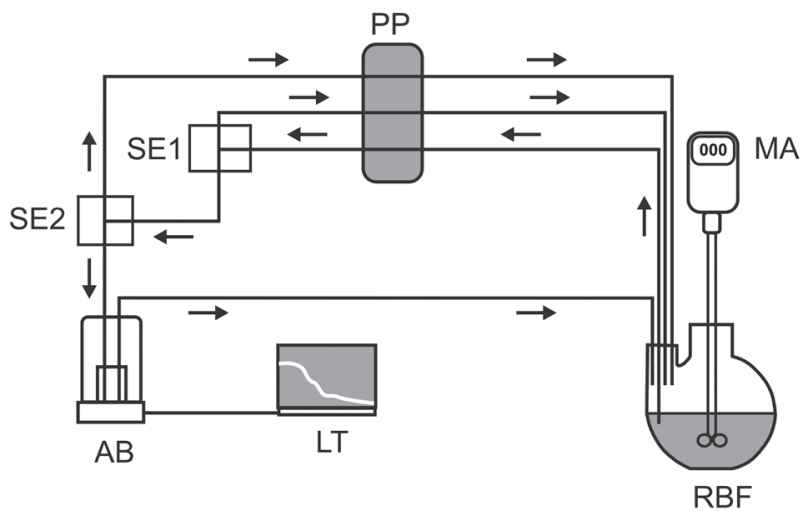

Figure 1. Schematic of the system used to monitor the transesterification reaction. RBF: round bottom flask where the reaction occurs; MA: mechanical stirrer; PP: peristaltic pump; SE1 and SE2: phase separators; AB: analytical balance; LT: laptop.

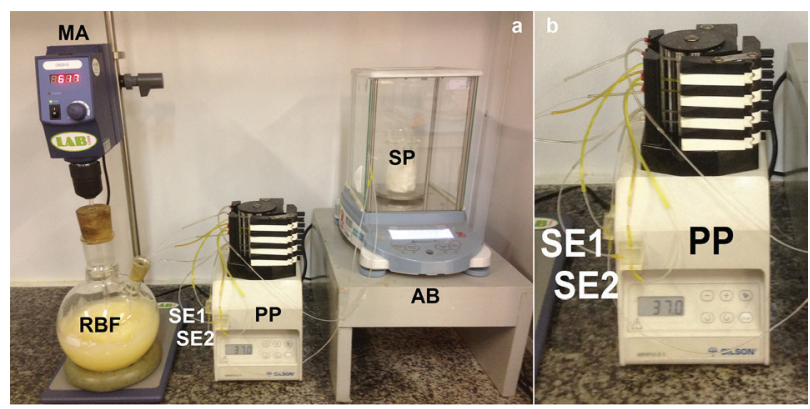

Figure 2. (a) Photo of the system detailed in Figure 1 without the laptop: MA: mechanical stirrer; RBF: round bottom flask; SE1 and SE2: phase separators; PP: peristaltic pump; AB: analytical balance; SP: polyethylene tube spiral; (b) enlarged portion from the photo in (a) showing the two phase separators.

\section{Monitoring the transesterification reaction}

For the transesterification reaction, $700.0 \mathrm{~g}$ of soybean oil, $120.0 \mathrm{~g}$ of methanol and $3.4 \mathrm{~g}$ of catalyst solution $(30 \%(\mathrm{~m} / \mathrm{v})$ sodium methoxide in methanol) were introduced into the round bottom flask. The mixture was stirred at $620 \mathrm{rpm}$ at $27^{\circ} \mathrm{C}$. The reaction was monitored over time, taking a reading every $30 \mathrm{~s}$. More details are provided in the Results and Discussion section below.

\section{Studies of the dispersion of methanol in soybean oil}

To observe the effect of stirring on the dispersion of methanol in oil, the system was assembled as described for the synthesis reaction except the catalyst that was omitted. The results are summarized in Figure 3.

In this study, the oil-methanol mixture was stirred at increasingly faster speeds, starting at $120 \mathrm{rpm}$ and

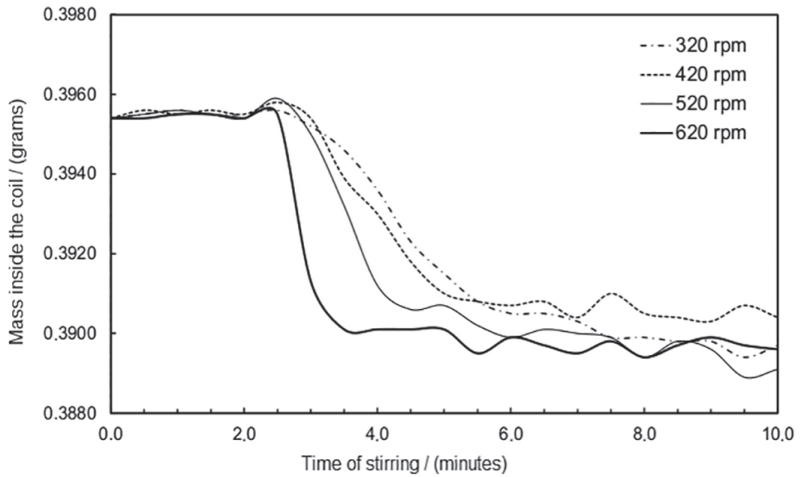

Figure 3. Formation of a dispersion of methanol in soybean oil. Variation of the mass inside the coil over time at different stirring rates at room temperature $\left(27^{\circ} \mathrm{C}\right)$.

increasing to $620 \mathrm{rpm}$ in $100 \mathrm{rpm}$ intervals. From 120 to $220 \mathrm{rpm}$, a dispersion of methanol in the oil was not formed. As a consequence, only oil was pumped to the analytical balance, which registered a constant value for the duration of the experiment.

Estimating the size of methanol drops dispersed in the soybean oil

Samples from the stirring oil-methanol mixture were collected using a polyethylene tube coupled to a hypodermic syringe. A drop of the mixture was immediately placed on a glass slide and covered with another slide to avoid evaporation of the methanol. These samples were observed and photographed using a Nikon E800 optical microscope with an Infinity 1 CCD camera. A Stage Micrometer Type A MBM11100 Nikon $0.01 \mathrm{~mm}$ was used to measure the diameter of methanol drops. To increase the contrast and ensure that the observed drops were not air bubbles, a commercial red food coloring was used.

Influence of temperature on the transesterification reaction

The influence of the temperature on the reaction rate was studied at $30.0,45.0$ and $60.0^{\circ} \mathrm{C}$. These results are summarized in Figures 4 and 5.

\section{Results and Discussion}

\section{Monitoring}

\section{Stabilization of the mass readings}

The assembled system was first tested by simply pumping vegetable oil from the round bottom flask at a flow rate of $1.1 \mathrm{~mL} \mathrm{~min}^{-1}$ while the oil was continuously stirring at $620 \mathrm{rpm}$. Monitoring was carried out until a maximum variation of $\pm 0.001 \mathrm{~g}$ was observed on the analytical 


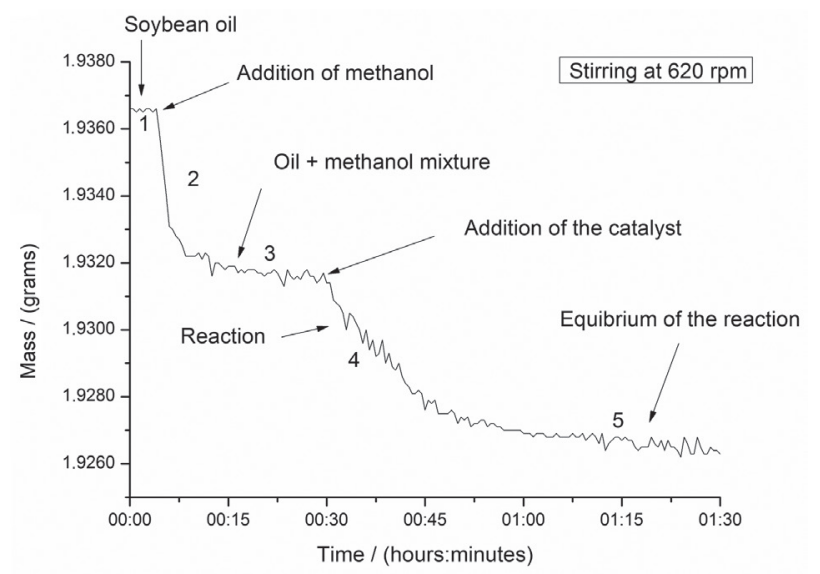

Figure 4. Mass of the transesterification reaction over time showing: (1) pure soybean oil; (2) dispersion of methanol in the oil; (3) equilibrium of the methanol + oil dispersion; (4) transesterification reaction; (5) equilibrium of the transesterification reaction. Reaction was carried out at room temperature $\left(27^{\circ} \mathrm{C}\right)$. The mixture was stirred at $620 \mathrm{rpm}$.

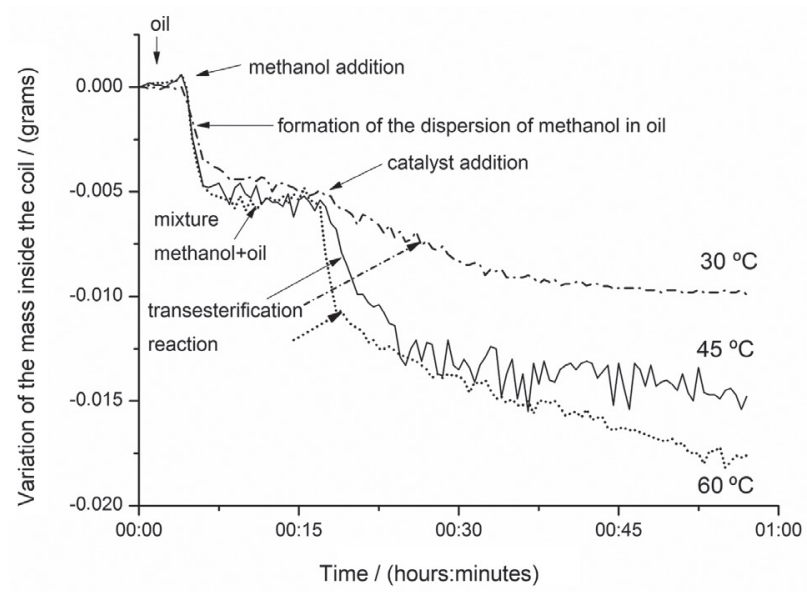

Figure 5. Transesterification reaction performed at three temperatures. Density of the oil + biodiesel mixture versus time. Reactions were performed at the indicated temperatures, but mass was measured at room temperature, ca. $27^{\circ} \mathrm{C}$. The mixture was stirred at $620 \mathrm{rpm}$.

balance. In cases where stability was not reached, the pump was turned off and the system was completely checked, especially the tube connections where leaking could occur.

\section{Dispersion of methanol in the soybean oil}

Methanol is not soluble in vegetable oils. Therefore, when this alcohol is used in the transesterification reaction, vigorous stirring is necessary to enhance the contact between these two reagents and also to increase contact with the catalyst, which is also dissolved in methanol. Figure 3 shows the variation in the density of the methanol + oil mixture as a function of time and at different stirring rates (at room temperature, $27{ }^{\circ} \mathrm{C}$ ). After about $8 \mathrm{~min}$ of stirring at $320 \mathrm{rpm}$, the system reached equilibrium; at higher stirring rates (420 or $520 \mathrm{rpm}$ ), equilibrium was achieved after about 5 or 6 min, leading to lower densities relative to stirring at slower speeds (320 rpm).
At $620 \mathrm{rpm}$, the equilibrium was reached after about $4 \mathrm{~min}$. The difference in densities achieved at different stirring rates indicates that when stirring at $320 \mathrm{rpm}$ the mixture does not attain maximal dispersion, while maximal dispersion is achieved at speeds of $420 \mathrm{rpm}$ or higher. Indeed, from 420 to $620 \mathrm{rpm}$ the density that the mixture attained reached approximately the same value, leading to a similar mass variation from the pure soybean oil to the mixture equilibrium.

The oscillation observed in the density measurements is likely a consequence of heterogeneity in the size of the methanol drops dispersed in the oil, a fact that leads to local changes in the density of the mixture.

Despite the fact that the final density of the mixture was independent of the stirring speed $(320,420,520$ or $620 \mathrm{rpm}$ ), it is clear from Figure 5 that the fluctuation in the reading is lowest at $620 \mathrm{rpm}$. Therefore, this rotation speed was used for all subsequent studies.

\section{Monitoring the transesterification reaction}

Soybean oil $(700.0 \mathrm{~g})$ was added to a $2 \mathrm{~L}$ round bottom flask equipped with a mechanical agitator rotating at $620 \mathrm{rpm}$. The peristaltic pump pushed the oil at a rate of $1.1 \mathrm{~mL} \mathrm{~min}{ }^{-1}$ through the network detailed in Figures 1 and 2 .

After stabilization of the reading on the balance, the system was monitored for about $5 \mathrm{~min}$ to be sure that the system was stable (Figure 4, region 1). Then $112 \mathrm{~g}$ of methanol were added causing a rapid decrease in the weight due to the lower density of the methanol + oil mixture relative to the oil alone (Figure 4, region 2). Equilibrium was reached when the mixture attained maximal dispersion, which is indicated by the lower density (Figure 4, region 3). Addition of $3.4 \mathrm{~g}$ of catalyst solution $(30 \%(\mathrm{~m} / \mathrm{v})$ of sodium methoxide in methanol) initiated the transesterification reaction and led to subsequent equilibrium (Figure 4, regions 4 and 5, respectively). The mixture was stirred at $620 \mathrm{rpm}$ and kept at room temperature $\left(27^{\circ} \mathrm{C}\right)$. The reaction was monitored over time and the mass was recorded every $30 \mathrm{~s}$.

The system was assembled according to the scheme shown in Figure 1. The liquid in the round bottom flask was continuously pumped from the surface in order to avoid pumping any glycerol, which is a byproduct of the transesterification reaction. The flow passed through two phase separators. The first phase separator, SE1, removed glycerol and air bubbles from the reaction mixture. The second separator, SE2, was placed in series to remove any residual glycerol and air bubbles. The liquid flowed into a spiral polyethylene tube that was placed in a beaker on the weighing pan of the analytical balance. This beaker contained a little piece of cotton that dampened the 
oscillations of the spiral during the experiment caused by the pulsing of the peristaltic pump. The mass was continuously monitored. As the volume in the spiral remained constant, changes in the density of the mixture led to changes in the mass reading. In the present case, as methyl fatty esters were formed during the transesterification reaction, the density decreased, leading to a decrease in the mass readings. Mass data were continuously recorded by the computer and displayed in a graph on the monitor.

The curves of mass variation (or density variation) in Figure 4 consisted of five parts, labeled 1 to 5 . Region 1 of the curve depends on the contents in the reaction flask, in this case soybean oil, which has a density of $0.914 \mathrm{~g} \mathrm{~cm}^{-3}$. The density of methanol is $0.792 \mathrm{~g} \mathrm{~cm}^{-3}$. Therefore a mixture of methanol + soybean oil has an intermediate density depending on the proportion of these two liquids, which was the cause for the mass decrease in part 2 of the curve. Part 3 signified stabilization of the mixture due to stirring. After addition of the catalyst, another decrease in the mass was observed in region 4 of the curve due to formation of the biodiesel, whose density is lower than the mixture of the two reagents. Finally, region 5 showed that the transesterification reaction reached equilibrium.

Mass was monitored until the reaction reached equilibrium and no more variation in the mass was observed. Frequently, at the end of the reaction there was a great quantity of glycerol present in the reaction flask, and a small amount of it reached the spiral positioned on the balance as the separators were not able to completely separate this phase. This caused important oscillations in the readings. Despite this, and also because of this instability, it became clear that the reaction had reached equilibrium.

Influence of temperature on the transesterification reaction

Figure 5 describes the behavior of the transesterification reaction at three different temperatures (30.0, 45.0 and $60.0^{\circ} \mathrm{C}$ ). It must be noted that the readings of the mass of the oil + biodiesel mixture contained in the coil on the pan of the analytical balance were performed at room temperature (ca. $27^{\circ} \mathrm{C}$ ). The profiles of the curves were similar at the three different temperatures as well as in the case depicted in Figure 4, where the reaction was performed at room temperature.

The first mass decrease observed was due to the addition of methanol to the oil. This assumption was supported by the fact that this decrease occurred similarly at all temperatures after addition of the methanol (Figure 5).

After adding catalyst to the reaction vessel, the decrease in mass over time occurred at different rates, with the slope becoming more negative as the temperature increased.
Temperature not only influenced the reaction rate, it also influenced the yield from the transesterification reaction. The lower density final product synthesized at higher temperatures indicated that a higher quantity of monoesters were formed.

Estimating the size of methanol drops in the oil + methanol mixture

Considering that the transesterification reaction occurs at the interface between the methanol and oil phases, ${ }^{11}$ it is useful to know the approximate size of the methanol drops in the mixture. It is known that the stirring frequency is a fundamental factor in the formation of dispersions. Stirring forms gradients in the velocity that generate the stress necessary for the comminution of methanol droplets. ${ }^{12}$ As drop size decreases, surface area increases, favoring the reaction.

Images obtained by electronic microscopy of the oil + methanol dispersion (without catalyst) obtained at $620 \mathrm{rpm}$ approached colloidal dimensions which by definition present sizes from 0.001 to $0.1 \mu \mathrm{m}$ in diameter. ${ }^{13}$ In Figure 6, it can easily be seen that a majority of the drops have a mean diameter of about $2 \mu \mathrm{m}$, signifying that the colloidal condition was almost achieved.

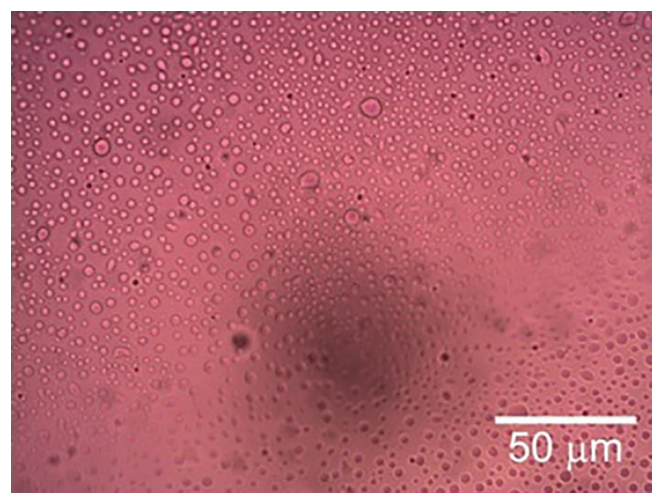

Figure 6. Microphotography of methanol drops in the oil + methanol mixture (without catalyst) taken with a NIKON E800 optical microscope with a $40 \times$ objective. The stage micrometer was a TYPE A MBM11100 Nikon $0.01 \mathrm{~mm}$. The mixture was stirred at $620 \mathrm{rpm}$.

Measurement of the drop size was performed on aliquots collected from the mixture stirring in the reaction flask and placed on a glass slide. Subsequently, another slide was placed on the first one containing the mixture. The photo was obtained about $40 \mathrm{~s}$ after sampling, which was required in order to adjust the focus of the camera. An increase in drop size during this time interval can be expected. Therefore, we estimate that at least some of the drops of methanol in the stirring reaction reach colloidal dimensions. 
We noted that during the time the methanol drops were on the slide they coalesced, leading to larger drop sizes. Because of this, the mixing system was placed next to the microscope, reducing the time interval between sampling and taking the photograph under the microscope to about $40 \mathrm{~s}$.

\section{Conclusions}

In light of the results obtained, we conclude that the proposed system is adequate for monitoring a transesterification reaction. Throughout the experiment clear signal changes were observed upon addition of methanol to the vegetable oil, during formation of the methanol + oil dispersion, and, after addition of the catalyst, the transesterification reaction from initiation to chemical equilibrium.

These experiments also demonstrate the importance of stirring the reaction mixture to form droplets of methanol with dimensions as close as possible to the colloidal condition in order to allow the transesterification reaction to occur.

Thus, based on these results, the proposed system can be used to monitor reactions of this type. This technique is quite useful as electronic analytical balances are inexpensive and ubiquitous in any chemical laboratory.

\section{Acknowledgments}

The authors would like to thank the Conselho Nacional de Ciência e Tecnologia (CNPq) for financial support and the Students Assistance Service (SAE) of the University of Campinas (UNICAMP) for the scholarship granted to A. G. O. J.

\section{References}

1. Demirbas, A.; Karslioglu, S.; Energy Convers. Manage. 2009 , $50,14$.

2. Demirbas, A.; Energy Policy 2007, 35, 4661.

3. Lôbo, I. P.; Ferreira, S. L. C.; Cruz, R. S.; Quim. Nova 2009 , 32, 1596.

4. Borsato, D.; Maia, E. C. R.; Dall'Antonia, L. H.; Silva, H. C.; Pereira, J. L.; Quim. Nova 2012, 35, 733.

5. Ghanei, R.; Moradi, G. R.; Taherpour Kalantari, R.; Arjmandzadeh, E.; Fuel Process. Technol. 2011, 92, 1593.

6. Arias, E. L. M.; Martins, P. F.; Monhoz, A. L. J.; GutierrezRivera, L.; Maciel Filho, R.; Ind. Eng. Chem. Res. 2012, 51, 10755.

7. Killner, M. H. M.; Rohwedder, J. J. R.; Pasquini, C.; Fuel 2011, 90, 3268.

8. Richard, R.; Li, Y.; Dubreuil, B.; Thiebaud-Roux, S.; Prat, L.; Bioresour. Technol. 2011, 102, 6702.

9. Tubino, M.; Rocha Junior, J. G.; Bauerfeldet, G. F.; Fuel 2014, 125, 164.

10. Tubino, M.; Rocha Junior, J. G.; Bauerfeldet, G. F.; Catal. Commun. 2016, 75, 6.

11. Kissa, E.; Dispersions, Characterization, Testing, and Measurement, Surfactant Science Series, vol. 84; Marcel Dekker: New York, USA, 1999, p. 18.

12. Morrison, I. D.; Ross, S.; Colloidal Dispersions - Suspensions, Emulsions and Foams; Wiley: New York, USA, 2002, p. 119.

13. Mingos, D.; Michael, P.; Gold Clusters, Colloids and Nanoparticles I; Springer: Oxford, United Kingdom, 2015, p. 5.

Submitted: March 17, 2017

Published online: June 26, 2017 